\title{
Raman spectroscopy setup and experiments for the advanced undergraduate lab
}

\section{Ralf Bausinger}

Ralf Bausinger, "Raman spectroscopy setup and experiments for the advanced undergraduate lab," Proc. SPIE 9793, Education and Training in Optics and Photonics: ETOP 2015, $97931 \mathrm{M}$ (8 October 2015); doi: $10.1117 / 12.2223121$

SPIE Event: Education and Training in Optics and Photonics: ETOP 2015, 2015, Bordeaux, France 


\title{
Raman Spectroscopy Setup and Experiments for the Advanced Undergraduate Lab
}

\author{
Ralf Bausinger \\ Universität Konstanz, Universitätsstraße 10, 78457 Konstanz, Germany
}

\begin{abstract}
Raman Spectroscopy is an important characterization technique in analytical chemistry and in solid state physics. This contribution describes the redesign of a Raman experiment for the Advanced Physics Lab and shows experimental results acquired with this new setup. Solid and liquid samples are irradiated by a frequency doubled Nd:YAG laser in backscattering geometry. The emission is focused onto the fibre end face of an USB spectrometer. Stokes and Anti-Stokes emission lines are observed simultaneously and their polarisation is analysed. The students start with the spectral calibration of the spectrometer and check its linearity. They optimize their first Raman spectrum of solid sulfur according to these results and evaluate the intensity ratio of the corresponding Stokes and Anti-Stokes lines for a temperature measurement of the sample. The students explain the spectral differences of a series of chlorine-substituted hydrocarbons by the changes in the molecular structures and symmetries. Finally they determine the concentration of an unknown water-ethanol solution. This contribution discusses also safety concerns regarding the used hydrocarbons and shows alternative samples. Raman Spectroscopy is an important characterization technique in analytical chemistry and in solid state physics. This contribution describes the redesign of a Raman experiment for the Advanced Physics Lab and shows experimental results acquired with this new setup. Solid and liquid samples are irradiated by a frequency doubled Nd:YAG laser in backscattering geometry. The emission is focused onto the fibre end face of an USB spectrometer. Stokes and Anti-Stokes emission lines are observed simultaneously and their polarisation is analysed. The students start with the spectral calibration of the spectrometer and check its linearity. They optimize their first Raman spectrum of solid sulfur according to these results and evaluate the intensity ratio of the corresponding Stokes and Anti-Stokes lines for a temperature measurement of the sample. The students explain the spectral differences of a series of chlorine-substituted hydrocarbons by the changes in the molecular structures and symmetries. Finally they determine the concentration of an unknown water-ethanol solution. This contribution discusses also safety concerns regarding the used hydrocarbons and shows alternative samples.
\end{abstract}

Keywords: Raman, Undergraduate Lab, Physics Education, Spectroscopy

\section{INTRODUCTION}

In Raman spectroscopy information about a sample is derived from the energy differences between incoming and scattered photons. Most photons are scattered elastically and conserve their energy (Rayleigh scattering). Approximately one of $10^{5}-10^{6}$ photons excites an internal degree of freedom of the sample and therefore looses part of its energy. This red-shifted photon is involved in a so called Stokes Raman process. An even smaller part of the photons undergoes an anti-Stokes Raman process and gains energy by de-exciting an internal vibration or oscillation. It is blue-shifted compared to the incoming photons. Since excitation and de-excitation bridge the same energy differences (Raman shifts), the resulting peak positions in the Raman spectrum are symmetric to the Rayleigh wavelength. The spectrum is given in wavenumber differences relative to the excitation wavelength, Stokes lines have positive and anti-Stokes lines negative Raman shifts. This not so common unit for the energy scale is often new for students but ensures that the characteristic Raman shifts for a specific sample are independent of the excitation wavelength. Since the intensities of the anti-Stokes lines are proportional to the number of already excited vibrations or rotations, they decrease exponentially with increasing Raman shift or inverse temperature. anti-Stokes intensities are reduced compared to the Stokes ones.

Raman spectroscopy is used for the structural analysis of molecules or solids and helps to identify substances by their characteristic fingerprint spectra. Its main application areas are analytical chemistry, pharmaceutical

Send correspondence to ralf.bausinger@uni-konstanz.de

Education and Training in Optics and Photonics: ETOP 2015, edited by Eric Cormier, Laurent Sarger Proc. of SPIE Vol. 9793, 97931M · @ 2015 SPIE, IEEE, OSA, ICO · doi: 10.1117/12.2223121 


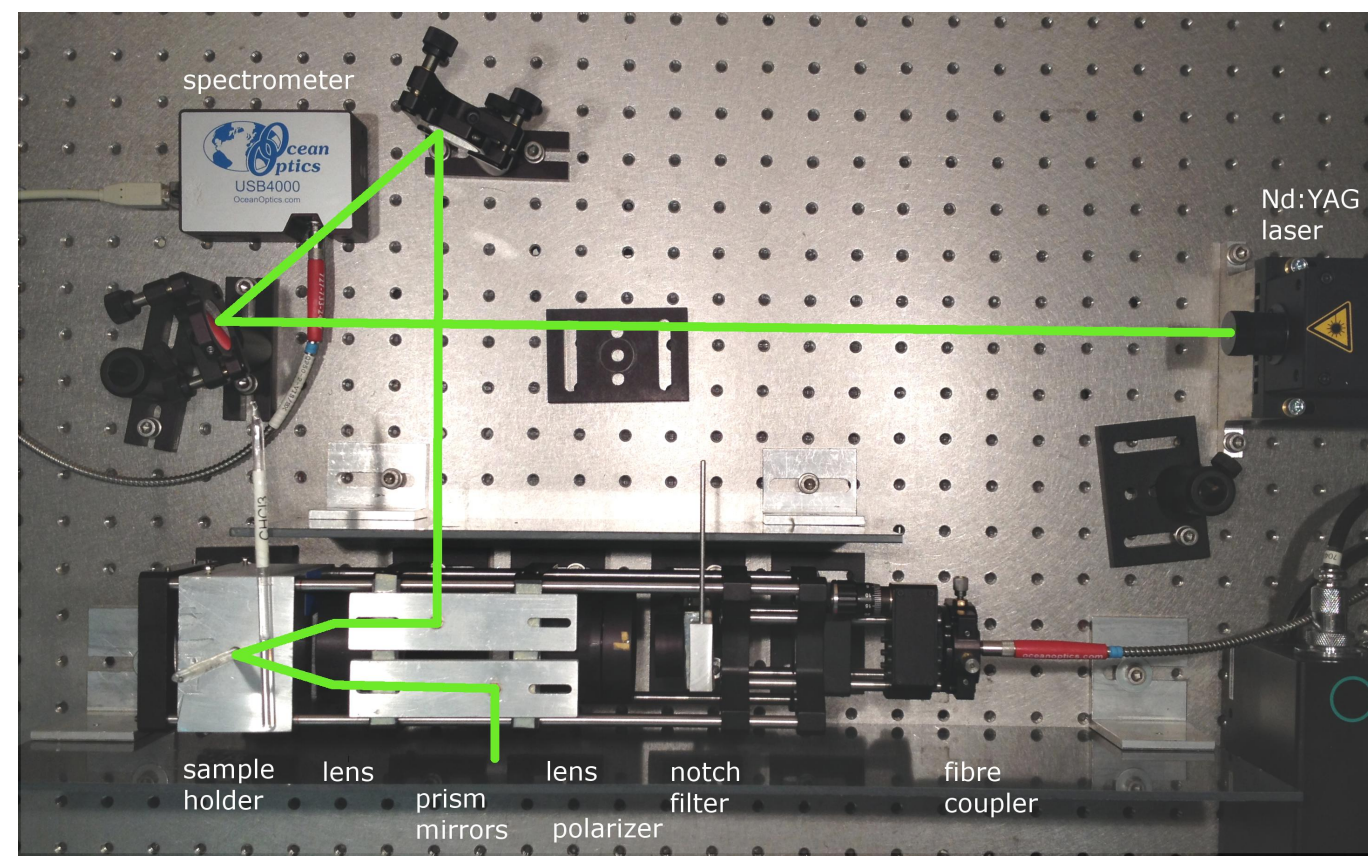

Figure 1. New Raman setup for the Advanced Physics Lab. Main parts are the Nd:YAG laser (right), the optical setup (bottom) with the sample on the left and the fibre on the right, coupling the light into the USB spectrometer (top left). The excitation beam path is sketched in green. Grid of holes in optical table is $25 \mathrm{~mm} \times 25 \mathrm{~mm}$.

and biomedical sciences, forensics, geology, material sciences, nanotechnology and solid state physics. The complementary technique to Raman spectroscopy for the investigation of vibrational and rotational excitations is IR absorption spectroscopy. It was a long time dominant over Raman spectroscopy until high intensity and narrow spectral linewidth light sources in form of lasers were available. Then Raman spectroscopy quickly became an important non-destructive analytical method, especially for aqueous systems. For these systems IR spectroscopy is not suitable due to the high infrared absorption of water.

\section{EXPERIMENTAL SETUP}

The two main parts of the old Raman spectroscopy setup in the Advanced Lab were a water cooled argon ion laser and a double prism spectrograph. The spectra were either acquired on Polaroid film and evaluated under a coordinate-reading microscope or recorded via a photomultiplier and fast NIM electronics on an xy pen plotter. It took around half an hour for the students to record an acceptable spectrum per sample and their focus was more on the technical part than on the spectral features they should observe. Therefore a new setup was designed with emphasize on the following criteria: short acquisition times of less than 1 minute, anti-Stokes Raman lines are observable, safe handling of volatile or hazardous samples, desktop size, less complicated components (no water cooling, no gas laser, less prone to failure, etc.)

The new setup is based on an air-cooled, frequency-doubled Nd:YAG laser at a wavelength of $532 \mathrm{~nm}$ and a small Czerny-Turner USB spectrometer. All the components fit on one half of an air-damped optical breadboard $(1 \mathrm{~m} \mathrm{x} 1.5 \mathrm{~m})$ shared with another experiment (wide-field and confocal fluorescence microscopy). Figure 1 shows the setup.

The laser beam is reflected by two mirrors for beam walking and by $90^{\circ}$ at a prism mirror and runs parallel to the optical axis of the Raman setup. An achromatic lens with a diameter and a focal length of $50 \mathrm{~mm}$ focuses the laser light onto the sample in the focal plane. Directly reflected excitation light hits a second prism mirror and is deflected off the optical axis into a beam dump. The solid or liquid samples are filled into NMR glass tubes with $5 \mathrm{~mm}$ diameter. These tubes can be fused at the top, so that volatile or hazardous substances are safely sealed within the vials. Around $1 \mathrm{~mL}$ of the sample substance is filled into the tube and temporarily frozen with liquid 
nitrogen. Then the top of the tube is melted with a Bunsen burner and the tube is sealed. The sample holder is an aluminium block with a vertical hole perpendicular to the optical axis for the NMR tube and a window for the laser excitation as well as the backwards scattered light. There is a threaded hole along the optical axis that allows for easy calibration with external lamps and which is blocked by a screw during Raman measurements.

The Raman light is emitted in all directions and part of it is collimated by the same lens which focuses the laser onto the sample. A second achromatic lens with a diameter of $50 \mathrm{~mm}$ and a focal length of $200 \mathrm{~mm}$ focuses the Raman light onto the end face of an optical fibre. Before hitting the fibre the converging light passes through a polariser and a notch filter. Usually these filters are placed in a section of the optical train with a collimated beam, because then all light from the focal spot hits the filter without inclination. This is relevant for interference filters, since their spectral characteristics depend on the angle of incidence. The notch filter in this setup has a diameter of $25 \mathrm{~mm}$ to save costs. It tolerates angles of incidence of up to $\pm 5^{\circ 1}$ and is therefore placed at a position in the optical path with a smaller beam diameter. The filter blocks light at (532 \pm 9$) \mathrm{nm}$ with an optical density of 6 . It is rotatable around a vertical axis so the angle of incidence and by that the blocked spectral region of the filter can be tuned. The polariser can be rotated around the optical axis to adjust for parallel or perpendicular polarisation relative to the vertically polarised excitation light. The position of the fibre end face can be adjusted in three dimension to optimize the coupling of the Raman light into the $50 \mu \mathrm{m}$ optical fibre. All the optical components are mounted in a cage system for $50 \mathrm{~mm}$ and $25 \mathrm{~mm}$ optics.

The fibre end face serves as input slit for the slitless USB spectrometer. The spectrometer was especially configured for the use in this Raman setup. The grating with 1800 lines/mm allows for a spectral range of $175 \mathrm{~nm}$, the start wavelength range was customized to start at $480 \mathrm{~nm}$, ranging up to $655 \mathrm{~nm}$. With an excitation wavelength of $532 \mathrm{~nm}$ this results in Raman shifts in the anti-Stokes region down below $-2000 \mathrm{~cm}^{-1}$ and up to $+3500 \mathrm{~cm}^{-1}$ in the Stokes region. In daily use the range on the Stokes side of the spectrum is adequate while anti-Stokes lines below $-1000 \mathrm{~cm}^{-1}$ were difficult to observe due to their small intensity. The CCD detector for the acquisition of the spectrum consists of 3648 elements. The sensitivity of the spectrometer was further enhanced by highly reflective mirrors and a collecting lens in front of the CCD detector array. The spectral data is send via USB interface to a personal computer and the spectrum can be viewed immediately on the monitor. This supports high quality spectra with optimized parameters, since the effect of changes can be observed and evaluated directly.

Concerning laser safety the excitation light is blocked by metal and plastics shields around the setup and the optical table. This prevents direct irradiation of the desktop working area and of neighbouring experiments. Additionally the students wear appropriate laser safety googles during the parts of the experiment with the laser turned on.

\section{EXPERIMENTS AND RESULTS}

The experiment starts with the characterisation of the spectrometer and in particular of the CCD detector. The dark current, its influence on the final spectrum and its dependence on the integration time are analysed. Students should conclude that they need to subtract a dark spectrum for every different acquisition time they use. As a next task students verify the spectral calibration of the spectrometer. They use helium and mercury lamps as light sources with different spectral lines. Other suitable calibration markers are the characteristic lines of mercury in fluorescent tubes or of neon in switch indicator lamps usually available on many devices in the lab. An important learning goal of this activity is the awareness that scientific instruments do not a priori deliver the 'exact result'. Finally detector saturation is analysed with a constant light intensity and increasing integration times.

The next unit deals with transmission and absorption spectra. As a practical application the students measure the transmission characteristics of the notch filter for different angles of incidence. Students and supervisor discuss the requirements and limitations for the acquisition of such a spectrum, as there are emission spectra of typical light sources, bright spectrum for normalization, digitisation and dynamic range, especially when measuring high optical densities like in the blocking region of the notch filter. With this knowledge they use an gooseneck halogen lamp as light source and acquire a dark spectrum, the emission spectrum of the lamp as a bright spectrum for normalization and finally the spectrum with the notch filter in the optical path. They observe the movement 


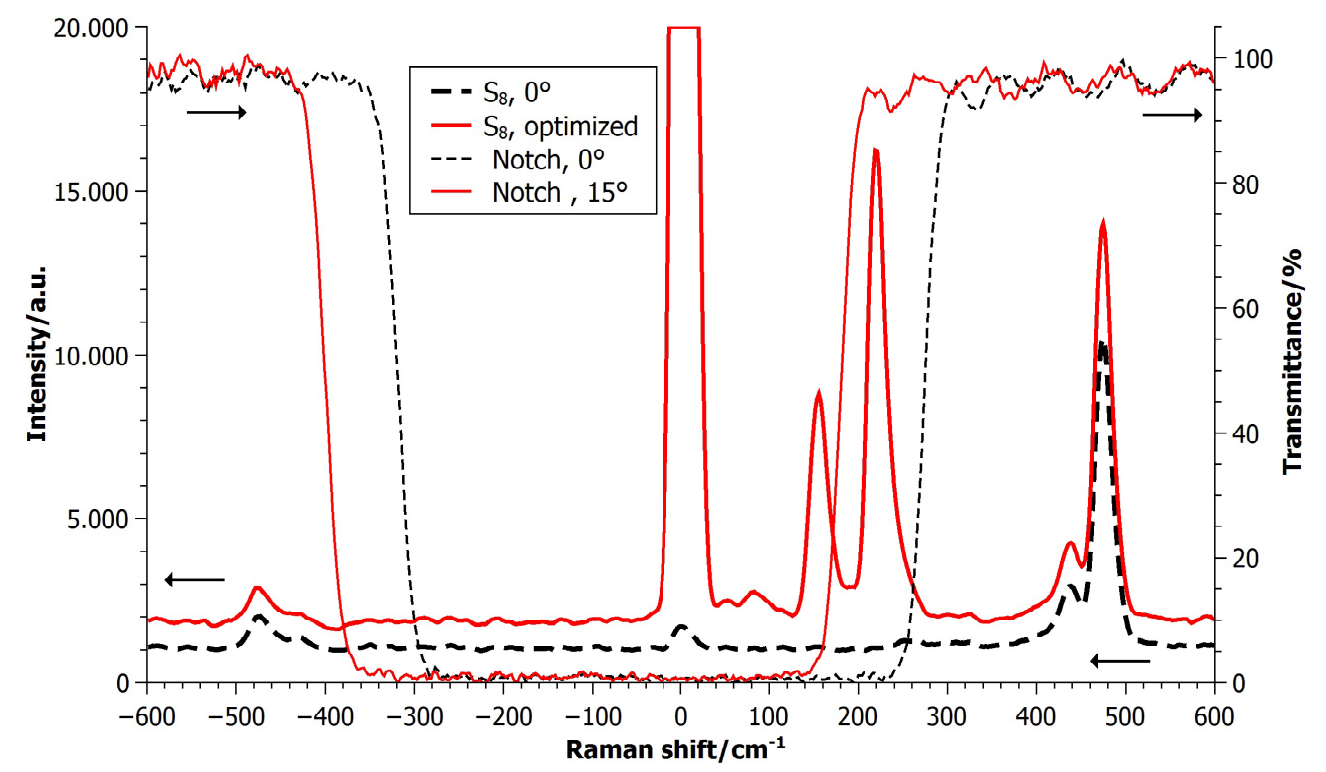

Figure 2. Sulfur Raman spectrum and notch filter transmittance. Raman spectra of sulfur (thick lines) show the effect of tilting the notch filter. At normal incidence (black, dashed) Raman lines below $300 \mathrm{~cm}^{-1}$ are obscured since the filter's transmittance (thin lines) is too low. A tilt of the filter by $15^{\circ}$ increases the transmission below $300 \mathrm{~cm}^{-1}$ (red solid line) and spectral features down to $50 \mathrm{~cm}^{-1}$ are observed with an optimized tilting angle (thick red solid line).

of the low transmission region towards shorter wavelengths when the angle of incidence is increased by turning the notch filter mount. Two typical transmission spectra for normal incidence and an angle of $15^{\circ}$ towards the normal are plotted in figure 2 .

This ability to tune the blocking region of the notch filter is important for the detection of Raman lines of low energy vibrations or rotations close to the Rayleigh peak. At normal incidence all Stokes lines with Raman shifts below $300 \mathrm{~cm}^{-1}$ are blocked by the notch filter. ${ }^{1}$ This is shown in the spectrum of pulverized sulfur $\left(\mathrm{S}_{8}\right)$ in figure 2, plotted as thick black dashed line. Solid sulfur is a good Raman scatterer and therefore ideally suited as a first sample for the students. With integration times around $300 \mathrm{~ms}$ adjustments of the acquisition parameters immediately show effects. By carefully tilting the notch filter the transmittance around the Rayleigh peak increases and further lines become visible. The thin red line in the figure shows the transmittance for $15^{\circ}$ angle of incidence. At the same time more remanent excitation and Rayleigh scattered light enters the spectrometer and finally saturates the detector. The central line at zero Raman shift appears broader and 'absorbs' nearby Raman lines. The students have to find a compromise between high transmittance for Raman lines and still sufficient attenuation at the excitation wavelength. The thick red line shows the sulfur spectrum for an optimized tilting angle of the notch filter. The intensity of the central Rayleigh peak is increased, but spectral features as close as $50 \mathrm{~cm}^{-1}$ to the central line are observed. Three additional spectral lines with maxima at $83 \mathrm{~cm}^{-1}, 155 \mathrm{~cm}^{-1}$ and $220 \mathrm{~cm}^{-1}$ become observable by fine-tuning the notch filter and are described in the literature. ${ }^{2}$ Related to the full width at half maximum of $30 \mathrm{~cm}^{-1}$ some individual features mentioned in the cited publication can not be resolved.

As a strong Raman scatterer with spectral features around $500 \mathrm{~cm}^{-1}$ sulfur clearly shows anti-Stokes lines which are analysed by the students for temperature measurements. Therefore the areas $S_{\mathrm{S}, \mathrm{AS}}$ under corresponding Stokes and anti-Stokes lines are measured and the ratio of these areas depends exponentially on the temperature of the sample: ${ }^{3}$

$$
\frac{S_{\mathrm{AS}}}{S_{\mathrm{S}}}=\left(\frac{\nu_{\mathrm{AS}}}{\nu_{\mathrm{S}}}\right)^{4} \exp \left(-\frac{h \nu_{\mathrm{P}}}{k_{\mathrm{B}} T}\right)
$$

$\nu_{\mathrm{AS}}$ and $\nu_{\mathrm{S}}$ are the frequencies of the scattered photons and $h \nu_{\mathrm{P}}$ is the energy difference between the excited and the ground state which is gained or lost by the anti-Stokes or Stokes photon in the scattering process. 


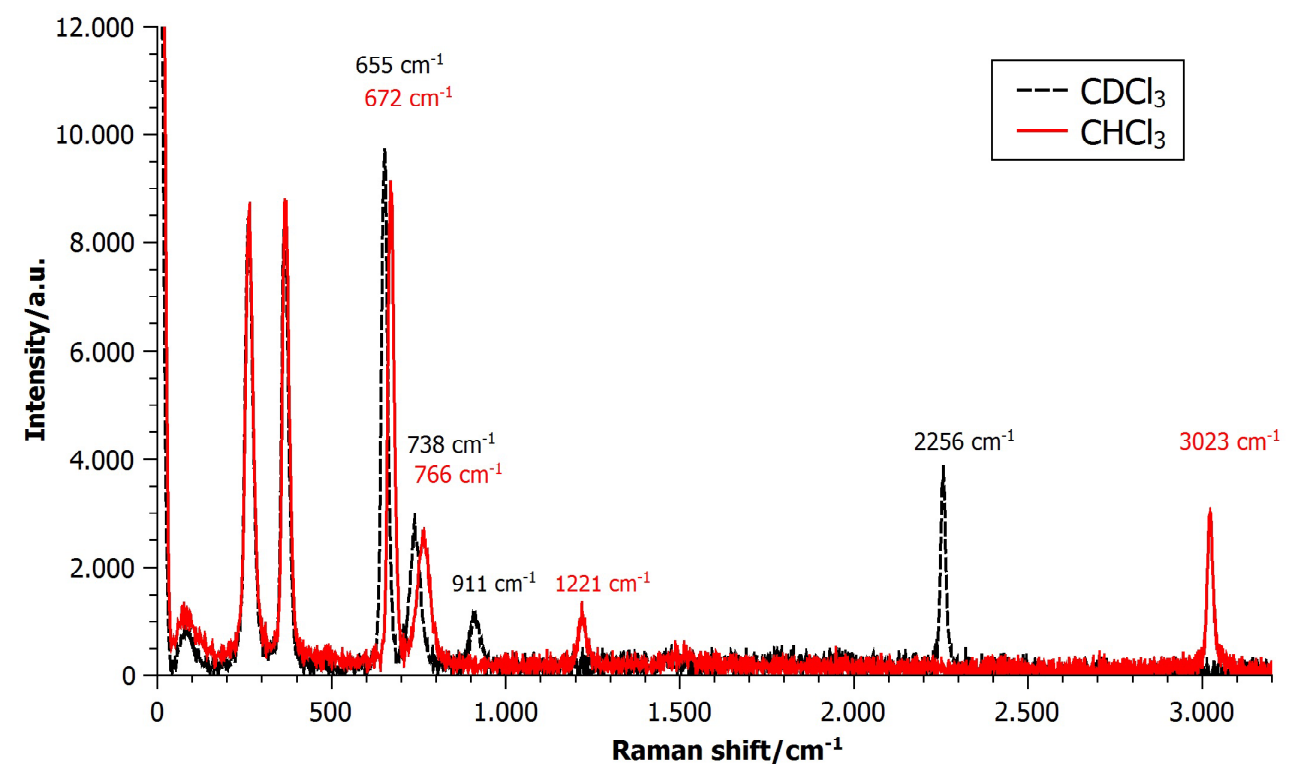

Figure 3. Raman spectrum of regular $\left(\mathrm{CHCl}_{3}\right.$, red solid line) and deuterated $\left(\mathrm{CDCl}_{3}\right.$, black dashed line) chloroform.

Students measure temperatures between $310 \mathrm{~K}$ and $330 \mathrm{~K}$. The elevated temperatures are related to laser heating of the sample and depend on the actual power of the laser.

Classical samples in Raman education are the chlorine substituted hydrocarbons $\mathrm{CH}_{2} \mathrm{Cl}_{2}, \mathrm{CHCl}_{3}$ and $\mathrm{CCl}_{4}$. Due to their low number in atoms and their clear symmetries their spectra can be analysed quite easily. Further information about the symmetry of individual vibrations can be gathered by polarisation dependent measurements. In the experiments in the Advanced Lab small quantities of these substances sealed in NMR tubes are used during the standard curriculum. Interested students can further explore oxyanions in saturated solutions of $\mathrm{Na}_{2} \mathrm{CO}_{3}, \mathrm{Na}_{2} \mathrm{SO}_{3}$ and $\mathrm{NaNO}_{3} \cdot{ }^{4}$

A valuable experience for students is the analysis of the line shifts between chloroform $\left(\mathrm{CHCl}_{3}\right)$ and the deuterated heavy form $\left(\mathrm{CDCl}_{3}\right)$. Figure 3 shows the Raman spectra of both substances. The task is to analyse the isotope shift of the spectral lines with the model of the diatomic oscillator. ${ }^{5}$ While most students can apply this model easily to the case of two atoms, they often have problems to explain the different ratios of the line positions just by grouping together different atoms. The low energy vibrations at $263 \mathrm{~cm}^{-1}$ and $366 \mathrm{~cm}^{-1}$ show no difference between the substances. This can be explained by the hypothesis that the three chlorine atoms and the hydrogen move as group. Then the change in reduced mass is negligible and the two lines are identical for both substances. For the next two line pairs at $655 / 672 \mathrm{~cm}^{-1}$ and $738 / 766 \mathrm{~cm}^{-1}$ the shift of the spectral lines is explained by the assumption that the hydrogen and the carbon atoms move as group against the chlorine atoms. The deuteration results in a change in reduced mass of $6 \%$, the Raman line is therefore shifted by $3 \%$. The largest shift is observed for the two high energy vibrations when the hydrogen oscillates against the group of carbon and chlorine atoms. It is very motivating for the students when they manage to apply this theoretical model to their own measurements and their predictions are in good agreement with their data.

The day in the lab ends with the determination of the ethanol concentration in an aqueous solution. ${ }^{6}$ Therefore the students are invited to bring a small sample of an alcoholic beverage to the lab session. After the identification of the characteristic Raman lines for ethanol and water the students calibrate their measurement with a dilution series of ethanol (96 vol.-\%) in water. When they try to analyse the ethanol/water ratio in their own sample they often find a strong fluorescence contribution in the spectrum due to additional substances like colourants or flavour additives. This is a good opportunity for the supervisor to give an outlook to more advanced Raman techniques like Resonance Raman Scattering or Surface-Enhanced Raman Scattering (SERS). 


\section{REFERENCES}

[1] Semrock Inc., "532 nm StopLine single-notch filter," tech. rep., Semrock Inc. (2015).

[2] Ward, A. T., "Crystal-field splitting of fundamentals in the raman spectrum of rhombic sulfur," The Journal of Physical Chemistry 72(2), 744-746 (1968).

[3] Wang, D., Mittauer, K., and Reynolds, N., "Raman scattering of carbon disulfide: The temperature effect," American Journal of Physics 77(12), 1130-1134 (2009).

[4] Comstock, M. G. and Gray, J. A., "Raman spectroscopy of symmetric oxyanions," Journal of chemical education 76(9), 1272 (1999).

[5] Wood, R. W. and Rank, D. H., "The raman spectrum of heavy chloroform," Phys. Rev. 48, 63-65 (Jul 1935).

[6] Sanford, C. L., Mantooth, B. A., and Jones, B. T., "Determination of ethanol in alcohol samples using a modular raman spectrometer," Journal of Chemical Education 78(9), 1221 (2001). 